\title{
Why helicity injection causes coronal flux tubes to develop an axially invariant cross-section
}

\author{
P. M. Bellan ${ }^{1}$ \\ ${ }^{1} M C$ 128-95, Caltech, Pasadena CA 91125, USA
}

\begin{abstract}
It is shown that electric current flowing along an axially non-uniform magnetic flux tube produces an associated non-linear, non-conservative axial MHD force which pumps plasma from regions where the flux tube diameter is small to regions where it is large. In particular, this force will ingest plasma into the ends of a fat, initially potential flux tube and then pump the ingested plasma towards the middle bulge, thereby causing mass accumulation at the bulge. The ingested plasma convects frozen-in toroidal magnetic flux which accumulates at the middle as well. Flux accumulation at the bulge has the remarkable consequence of causing the bulge to diminish so that the flux tube becomes axially uniform as observed in coronal loops. Stagnation of the convergent plasma flow at the middle heats the plasma. A small number of tail particles bouncing synchronously between approaching fluid elements can be Fermi-accelerated to very high energies. Since driving a current along a flux tube is tantamount to helicity injection into the flux tube, this mass ingestion, heating, and straightening should be ubiquitous to helicity injection processes.
\end{abstract}

\section{Introduction}

Remarkably detailed images of coronal loops provided by the TRACE spacecraft [Aschwanden et al (2000)] indicate that these loops have cross-sectional area varying by only 10-20\% over their entire length; this surprising behavior cannot be explained by potential or force-free field models [Klimchuk (2000]. We present here a model explaining this behavior. Since the loops are typically twisted by a fraction of a turn over their length, they contain magnetic helicity and so our model should be intrinsic to helicity injection processes.

A coronal loop will be represented here by an axisymmetric flux tube with straight axis (cf. top, Fig. 1) and cylindrical coordinates $(r, \phi, z)$ will be used. The loop is characterized by an initially potential poloidal flux function

$$
\psi(r, z)=\int_{0}^{r} B_{z}\left(r^{\prime}, z\right) 2 \pi r^{\prime} d r^{\prime}
$$

with associated poloidal field

$$
\mathbf{B}_{p o l}=\frac{1}{2 \pi} \nabla \psi \times \nabla \phi .
$$

Axial non-uniformity corresponds to $\psi$ being $z$-dependent and bulging corresponds to $\psi^{-1} \partial^{2} \psi / \partial z^{2}>0$. Similarly, the poloidal current is written as

$$
I(r, z)=\int_{0}^{r} J_{z}\left(r^{\prime}, z\right) 2 \pi r^{\prime} d r^{\prime}
$$

with associated poloidal current density

$$
\mathbf{J}_{p o l}=\frac{1}{2 \pi} \nabla I \times \nabla \phi .
$$


We show that establishing a steady-state current $I$ involves three sequential stages having distinct physics. The first stage, "ramp-up", has physics akin to a linear Alfvén wave, but it is assumed that the ramp-up rate is sufficiently slow that the effective Alfvén wavelength is infinite. This means that retarded time effects due to wave propagation issues are negligible and the current ramps up everywhere simultaneously as in an ordinary electrical circuit. The second stage, "axial flow", has $\partial I / \partial t=0$, but is not in MHD equilibrium because unbalanced, non-conservative $\mathbf{J} \times \mathbf{B}$ forces exist which drive plasma flows. The third stage, "stagnation", involves convection of magnetic flux by the flows, plasma heating as a result of flow stagnation, and straightening of the $\psi$ profile until MHD equilibrium is established.

\section{First stage (Ramp-up)}

We represent the current ramp-up by the time-dependence

$$
I(t)=I_{0} \frac{1+\tanh (t / \tau)}{2}
$$

where the ramp-up time is assumed to be much longer than the time it takes for an Alfvén wave to propagate the length $h$ of the flux tube, i.e., $\tau>>h / v_{A}$. Alfvén wave propagation effects are therefore unimportant in which case the system behaves like an electric circuit. From Ampere's law the toroidal magnetic field is

$$
B_{\phi}(r, z, t)=\frac{\mu_{0} I(r, z, t)}{2 \pi r} .
$$

The toroidal component of Faraday's law is

$$
\frac{\partial E_{r}}{\partial z}-\frac{\partial E_{z}}{\partial r}=-\frac{\partial B_{\phi}}{\partial t}
$$

We note that $B_{\phi}$ has minimal $z$ dependence and that Ohm's law implies $E_{z} \simeq 0$. Thus, integration of Eq.(17) with respect to $z$ gives

$$
E_{r} \simeq-\frac{\mu z}{2 \pi r} \frac{\partial I}{\partial t}
$$

where on the basis of symmetry the location $z=0$ is set to be at the axial midpoint of the flux tube. Since there is no axial force in this stage, $U_{z}$ remains zero and so the radial component of the ideal Ohm's law gives

$$
U_{\phi}=-\frac{E_{r}}{B_{z}} \simeq \frac{\mu z}{2 \pi r B_{z}} \frac{\partial I}{\partial t},
$$

showing that $U_{\phi}$ is finite only when $I$ is changing. The change in $U_{\phi}$ (toroidal acceleration) implies the existence of a radial current determined from the toroidal component of the equation of motion

$$
\rho \frac{\partial U_{\phi}}{\partial t}=-J_{r} B_{z}
$$

This current is just the polarization current [Chen(1984), p.40]

$$
J_{r}=-\frac{\rho}{B_{z}} \frac{\partial U_{\phi}}{\partial t}=\frac{\rho}{B_{z}^{2}} \frac{\partial E_{r}}{\partial t}=-\frac{\mu z \rho}{2 \pi r B_{z}^{2}} \frac{\partial^{2} I}{\partial t^{2}} .
$$

The transient toroidal velocity given by Eq.(9) results in an azimuthal displacement of the plasma,

$$
r \Delta \phi=\int_{0}^{t} U_{\phi} d t=\frac{\mu_{0} z I}{2 \pi r B_{z}}=\frac{z B_{\phi}}{B_{z}}
$$

showing that the plasma motion in this stage follows the twisting of the magnetic field (the field line can be thought of as being frozen to the plasma so that when the field line twists, so does the plasma). Thus 


\section{Initially untwisted potential flux loop}

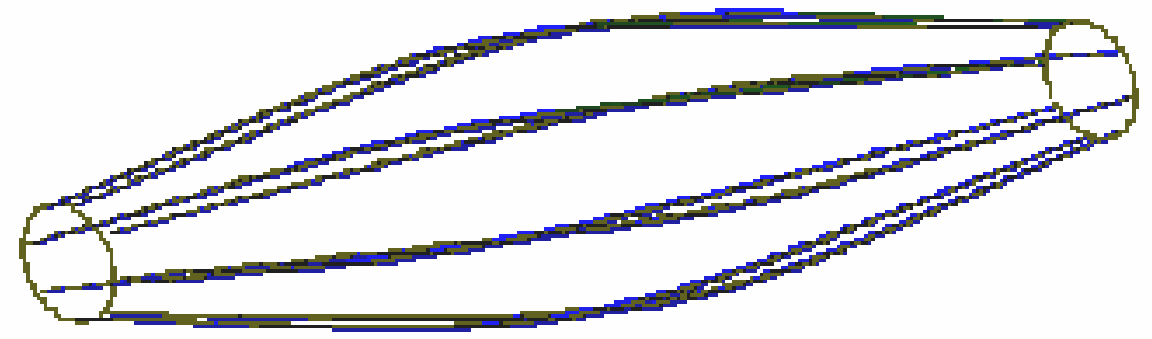

\section{Axial current twists flux loop}

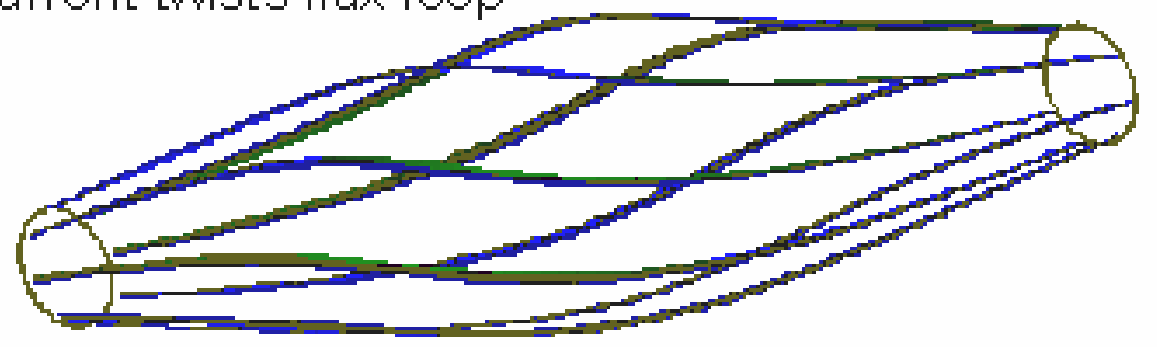

Fig. 1. Top: Initially potential flux tube. Bottom: Flux tube with axial current has same poloidal profile, but is twisted. Cylindrical geometry $(r, \phi, z)$ is used; $z=0$ is the flux tube axial midpoint and the flux tube ends are at $z= \pm h$.

$J_{r}$ is finite only when $I$ is changing and $J_{r}$ is first negative and then positive, corresponding to toroidal acceleration followed by toroidal deceleration. The $r$ direction here is really a proxy for the $\nabla \psi$ direction, since the polarization current is in the $\nabla \psi$ direction (because the flux tube is long and slender, the $r$ direction is nearly the same as the $\nabla \psi$ direction).

Once $I$ has been established, both $U_{\phi}$ and the polarization current $J_{r}$ remain zero. The poloidal flux function $\psi$ at this stage has not changed from its original value - all that has happened is that a toroidal field has been added so that the total magnetic field is now

$$
\mathbf{B}=\frac{1}{2 \pi}\left(\nabla \psi \times \nabla \phi+\mu_{0} I \nabla \phi\right) .
$$

The original potential flux tube has become twisted as shown in the bottom sketch of Fig. 1. Since $\psi$ is unchanged from its potential value, the bottom sketch in Fig.1 has the same poloidal profile (envelope) as the top sketch. This means that

$$
\mu_{0} J_{\phi}=r \nabla \phi \cdot \nabla \times \frac{(\nabla \psi \times \nabla \phi)}{2 \pi}=-\frac{r}{2 \pi} \nabla \cdot\left(\frac{1}{r^{2}} \nabla \psi\right)=0 .
$$




\section{Second stage (flow acceleration)}

The magnetic force is

$$
\begin{aligned}
\mathbf{J} \times \mathbf{B} & =\left(\mathbf{J}_{p o l}+\mathbf{J}_{t o r}\right) \times\left(\mathbf{B}_{p o l}+\mathbf{B}_{t o r}\right) \\
& =\mathbf{J}_{p o l} \times \mathbf{B}_{\text {pol }}+\mathbf{J}_{p o l} \times \mathbf{B}_{t o r}+\mathbf{J}_{t o r} \times \mathbf{B}_{p o l} .
\end{aligned}
$$

The term $\mathbf{J}_{p o l} \times \mathbf{B}_{p o l}$ provides acceleration in the toroidal direction and involves the component of $\mathbf{J}_{p o l}$ which is perpendicular to $\mathbf{B}_{p o l}$. However, in the previous section we showed that the component of $\mathbf{J}_{\text {pol }}$ which is perpendicular to $\mathbf{B}_{p o l}$ is the polarization current and that this current scales as $\partial^{2} I / \partial t^{2}$. Thus, when $I$ is in steady state there is no polarization current and no toroidal acceleration, and so $U_{\phi}$ remains zero. It was also shown that $\mathbf{J}_{t o r}=J_{\phi} \hat{\phi}$ is zero so long as the poloidal flux surfaces are unperturbed from their initial potential shape. We assume in this second stage that (i) the current is constant in which case $\mathbf{J}_{\text {pol }} \times \mathbf{B}_{\text {pol }}=0$ and $U_{\phi}=0$ and (ii) the poloidal flux surfaces are unperturbed from their initial potential shape in which case $\mathbf{J}_{\text {tor }}=0$. Thus, during this second stage the magnetic force reduces to

$$
\mathbf{J} \times \mathbf{B}=\mathbf{J}_{p o l} \times \mathbf{B}_{t o r}=\frac{1}{2 \pi}(\nabla \psi \times \nabla \phi) \times \frac{\mu_{0} I}{2 \pi} \nabla \phi=-\frac{\mu_{0}}{8 \pi^{2} r^{2}} \nabla I^{2} .
$$

The above force is non-conservative (i.e., $\nabla \times(\mathbf{J} \times \mathbf{B})$ is non-zero) and so cannot be balanced by a pressure gradient since a pressure gradient is conservative (i.e., $\nabla \times \nabla P=0$ ). Thus, it is not possible for equilibrium to be attained in this stage. The only way for an equilibrium to be achieved is for the poloidal profile of the magnetic field to change, which is what happens in the third stage, to be discussed later.

The fact that $\mathbf{J}_{p o l} \times \mathbf{B}_{\text {pol }}=0$ means that $(\nabla I \times \nabla \phi) \times(\nabla \psi \times \nabla \phi)=0$ which in turn implies that $\nabla I$ is parallel to $\nabla \psi$ and so $I$ must be a function of $\psi$, i.e., $I=I(\psi)$. Thus, the poloidal current flows along the poloidal flux surfaces. This is consistent with the well-known Hamiltonian dynamics concept that, because of conservation of canonical angular momentum, particles in a toroidally symmetric geometry cannot make an excursion exceeding a poloidal Larmor radius from a poloidal flux surface [e.g., see p.207-208 of Bellan(2000)]. In other words, Hamiltonian mechanics forbids the existence of steady current in the direction normal to a poloidal flux surface.

As sketched in Fig. 1, the poloidal flux function is bulged near $z=0$, corresponding to a weaker magnetic field near $z=0$ than at the ends $z= \pm h$. This would be the situation if the source currents for the poloidal field were located external to the flux tube and so the middle of the flux tube would be further from the source currents than the ends. Since $I=I(\psi)$, the current channel would also be bulged.

Equation (16) implies that the $z$ component of the equation of motion is

$$
\rho \frac{d U_{z}}{d t}=(\mathbf{J} \times \mathbf{B})_{z}=-\frac{1}{8 \pi^{2} r^{2}} \frac{\partial I^{2}}{\partial z}=-\frac{\partial}{\partial z}\left(\frac{B_{\phi}^{2}}{2 \mu_{0}}\right) .
$$

This means that there is a force accelerating plasma from regions where $B_{\phi}^{2}$ is strong to regions where $B_{\phi}^{2}$ is weak. Since $I=I(\psi)$ and since $\psi$ is bulged in the middle, $B_{\phi}$ must be stronger near $z= \pm h$ where the current channel diameter is small than at $z=0$ where the current channel diameter is large. There consequently must be an acceleration of plasma from both ends (i.e., $z= \pm h$ ) towards the middle (i.e., $z=0$ ) as shown in Fig. 2. The convergent axial pumping is similar to the "sweeping magnetic twist mechanism" discussed by Uchida and Shibata (1988), but it should not be considered a wave because it involves actual convection of material and not propagation of energy through a material. We note in passing that there could be a few exceptional particles collisionally bouncing back and forth between the approaching fluid elements. These exceptional particles would be accelerated to very high energy by the Fermi acceleration process, and so one would expect to see a tail of energetic particles develop in the vicinity of $z=0$. The Fermi process would thus predict that the most energetic particles would be located around the top of an arched coronal loop and such is indeed what is observed (Feldman, 2002). 


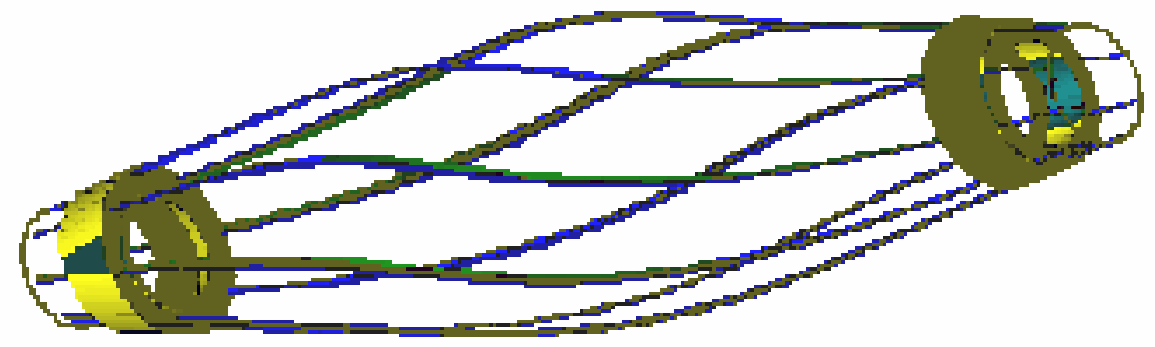

Fig. 2. Toroidal plasma fluid elements are accelerated from $z \pm h$ to $z=0$ by force $F_{z}=-\partial\left(B_{\phi}^{2} / 2 \mu_{0}\right) / \partial z$. These representative fluid elements move towards each other, but do not rotate.

\section{Third stage (stagnation, heating, and straightening)}

The flows from both ends converge at the middle and must come to a halt at $z=0$. Convergence of flows means that $\nabla \cdot \mathbf{U}$ is negative. This has important implications for the magnetic field as can be seen by considering the induction equation toroidal component,

$$
\frac{\partial B_{\phi}}{\partial t}=r \mathbf{B}_{p o l} \cdot \nabla\left(\frac{U_{\phi}}{r}\right)-r \mathbf{U}_{p o l} \cdot \nabla\left(\frac{B_{\phi}}{r}\right)-B_{\phi} \nabla \cdot \mathbf{U}_{p o l} .
$$

We have shown that (i) $U_{\phi}=0$, (ii) $\mathbf{U}_{p o l} \rightarrow 0$ at the stagnation layer, and (iii) $\nabla \cdot \mathbf{U}_{\text {pol }}$ is negative. Thus, in the vicinity of the stagnation layer

$$
\frac{\partial B_{\phi}}{\partial t}=-B_{\phi} \nabla \cdot \mathbf{U}_{p o l}
$$

showing that $B_{\phi}$ must increase at the stagnation layer (increase of magnetic field at regions of local flow convergence has been discussed in a more general context by Polygiannakis and Moussas (1999)).

The continuity equation in the vicinity of the stagnation layer gives $\nabla \cdot \mathbf{U}_{p o l}=-\rho^{-1} \partial \rho / \partial t$ and so in the vicinity of the stagnation layer

$$
\frac{1}{B_{\phi}} \frac{\partial B_{\phi}}{\partial t}=\frac{1}{\rho} \frac{\partial \rho}{\partial t}
$$

Thus, in the vicinity of the stagnation layer $B_{\phi}$ increases in proportion to the accumulation of mass. Since $I$ is constant during this stage, if $B_{\phi}$ increases, the radius of the current channel must decrease so as to maintain $\mu_{0} I=2 \pi r B_{\phi}$ constant. However, since $I=I(\psi)$, if the radius of the current channel decreases, then the radius of $\psi$ must also decrease, thereby reducing the bulging. Ultimately, the bulge becomes vanishingly small as more and more mass accumulates at the stagnation point and eventually a plasma-loaded, axially uniform flux tube results.

Since stagnation involves conversion of flow velocity into thermal energy, the plasma will be heated at the stagnation layer. The resulting plasma temperature can be estimated from the details of the stagnation process. The axial magnetic force in Eq.(17) involves the gradient of the toroidal field energy density and so the kinetic energy gained by the plasma will be of the order of the change in toroidal field energy density 
$B_{\phi}^{2} / 2 \mu_{0}$ between the ends $(z= \pm h)$ and the midpoint $z=0$. Because $B_{\phi}^{2} / 2 \mu_{0}$ is larger at the ends, the plasma flow kinetic energy is $\rho U_{z}^{2} / 2 \simeq\left[B_{\phi}^{2} / 2 \mu_{0}\right]_{z= \pm h}$ and at the stagnation layer $z=0$ this flow energy is converted into heat so $\rho U_{z}^{2}=\left[B_{\phi}^{2} / \mu_{0}\right]_{z= \pm h} \rightarrow n m_{e} v_{T e}^{2}+n m_{i} v_{T i}^{2}$.

Since the poloidal field is much stronger than the toroidal field and is approximately $B_{z}$, this means that the flow stagnation causes the plasma to develop a state where

$$
\beta=\frac{2 \mu_{0}\left(n m_{e} v_{T e}^{2}+n m_{i} v_{T i}^{2}\right)}{B_{z}^{2}}=2 \frac{B_{\phi}^{2}}{B_{z}^{2}}=2\left(\frac{\mu_{0} I}{2 \pi a B_{z}}\right)^{2}=\left(\frac{\mu_{0} I}{\psi}\right)^{2} \frac{a^{2}}{2},
$$

where $a$ is the radius of the current channel. However, $\mu_{0} I=\int d \mathbf{s} \cdot \nabla \times \mathbf{B}$ and $\psi=\int d \mathbf{s} \cdot \mathbf{B}$ where the surface integral is over the cross-sectional area of the flux tube. If we define the ratio of poloidal current to poloidal flux as

$$
\alpha=\mu_{0} I / \psi
$$

the $\beta$ predicted from flow stagnation is

$$
\beta=\alpha^{2} a^{2} / 2 .
$$

Thus, when a current is made to flow along an initially bulging current-free current channel, the current channel will twist up (helicity injection), plasma will be ingested from both ends, accelerated toward the middle where it accumulates and heats up at a stagnation layer. The equilibrium will become straight (filamentary) and have $\beta=\alpha^{2} a^{2} / 2$ where $\alpha=\mu_{0} I / \psi$ and $a$ is the radius of the current channel. To an outside observer the current channel will look field-aligned since the current is axially uniform and appears to be embedded in an axially uniform axial magnetic field. However, oblivious to the outside observer, within the current channel there is a Bennett pinch-like radial force balance between plasma pressure pushing out and $J_{z} B_{\phi}$ magnetic force pushing in.

Although the predicted $\beta$ is typically very small, its effect is crucial. To see this, consider that in equilibrium $\mathbf{J} \times \mathbf{B}=\nabla P$ so that $\mathbf{B} \cdot \nabla P=(2 \pi)^{-1}\left(\nabla \psi \times \nabla \phi+\mu_{0} I \nabla \phi\right) \cdot \nabla P=0$ and so $P$ must be a function of $\psi$, i.e., $P=P(\psi)$. Defining $\psi_{0}$ as the flux on the flux surface where $P$ vanishes, we can write $P(r, z)=\left(1-\psi(r, z) / \psi_{0}\right) P_{0}$ where $P_{0}$ is the on-axis pressure (i.e., where $\left.\psi=0\right)$. We can also write $\mu_{0} I(r, z)=\alpha \psi(r, z)$ and so $\mathbf{J} \times \mathbf{B}=\nabla P$ can be written in Grad-Shafranov form as

$$
r \frac{\partial}{\partial r}\left(\frac{1}{r} \frac{\partial \psi}{\partial r}\right)+\frac{\partial^{2} \psi}{\partial z^{2}}+\alpha^{2} \psi=4 \pi^{2} \mu_{0} r^{2} \frac{P_{0}}{\psi_{0}} .
$$

If $\alpha^{2}=4 \pi^{2} \mu_{0} a^{2} P_{0} / \psi_{0}^{2}$ where $a$ is the flux tube radius at $z=0$, then the only solution to Eq.(24) satisfying the specified boundary condition that $P$ vanishes when $\psi=\psi_{0}$ is the particular solution $\psi(r, z)=\psi_{0} r^{2} / a^{2}$. This means that the flux tube must be axially uniform when $\alpha^{2}=4 \pi^{2} \mu_{0} a^{2} P_{0} / \psi_{0}^{2}$. Defining $B_{0}=\psi_{0} / \pi a^{2}$ as the axial field at $z=0$, it is seen that this condition for axial uniformity corresponds to $\alpha^{2}=4 \pi^{2} \mu_{0} a^{2} P_{0} / B_{0}^{2} \pi^{2} a^{4}$ or $\alpha^{2} a^{2} / 2=\beta$ where $\beta=2 \mu_{0} P_{0} / B_{0}^{2}$. This equilibrium has $J_{\phi}=0$, so all confinement is provided by the Bennett pinch force $\sim J_{z} B_{\phi}$. The current is purely in the $z$ direction, but the magnetic field is helical.

The situation of small but finite $\beta$ is substantially different from the case of zero $\beta$ because the system is constrained to be axially uniform if and only if $\beta=\alpha^{2} a^{2} / 2$. The arguments presented in the discussion of Eqs.21-23) show that the MHD dynamical pumping tends to produce precisely the situation where $\beta=\alpha^{2} a^{2} / 2$, and so it is predicted that MHD dynamical pumping will always cause configurations to tend towards being axially uniform (i.e., filamentary), hot, and dense, and with $\beta=\alpha^{2} a^{2} / 2$.

The definition of $\alpha$ in Eq.(22) is closely related to that used for force-free fields. However, there is an important difference because while Eq.(22) corresponds to having $\mu_{o} J_{z}=\alpha B_{z}$, Eq. (22) makes no statement about any relationship between $J_{\phi}$ and $B_{\phi}$. The finite $\beta$ equilibrium discussed in the previous two paragraphs is not force-free and involves the radial force balance $J_{z} B_{\phi}=-\partial P / \partial r$ with $J_{\phi}=0$; this differs from the force-free radial equation $J_{\phi} B_{z}-J_{z} B_{\phi}=0$ with $\mu_{o} J_{\phi}=\alpha B_{\phi}, \mu_{o} J_{z}=\alpha B_{z}$. It is worth noting that the determination of $\alpha$ made from vector magnetographs (e.g., Pevtsov et al., 1997) effectively use the definition 
$\alpha=\mu_{o} J_{z} / B_{z}$ which is equivalent to Eq.(22); these measurements do not provide information on either $J_{\phi}$ or $B_{\phi}$ and so do not provide any information on the value of $\mu_{o} J_{\phi} / B_{\phi}$. Thus there is only one definition for $\alpha$, but its application is different for force-free situations compared to finite- $\beta$ situations: for force-free situations $\alpha$ gives the ratio of current to flux for both toroidal and poloidal directions whereas for the finite $\beta$ situation, $\alpha$ refers only to the the ratio of poloidal current to poloidal field.

The prediction that $\beta=\alpha^{2} a^{2} / 2$ can be compared with the actual observed values of $\beta$ in TRACE flux loops. To calculate the predicted $\beta$, we use the nominal measured flux loop radius $a=1.6 \times 10^{6} \mathrm{~m}$ from Aschwanden et al.(2000) and the nominal measured active region $\alpha=2 \times 10^{-8} \mathrm{~m}^{-1}$ from Fig. 4 of Pevtsov et al. (1997). These parameters give a nominal $\beta_{\text {predicted }}=\alpha^{2} a^{2} / 2=5 \times 10^{-4}$. The observed value $\beta_{\text {observed }}$ is calculated using a nominal measured density $n=10^{15} \mathrm{~m}^{-3}$ and a nominal measured temperature $10^{6}$ $\mathrm{K}$ [Aschwanden et al. (2000)]. In addition a nominal axial magnetic field $B_{z}=1.5 \times 10^{-2} \mathrm{~T}$ is assumed based on the argument that because the flux tube is axially uniform, its axial field must also be axially uniform and so will have the same value as the nominal $B_{z}=1.5 \times 10^{-2}$ at the surface of an active region. These parameters give $\beta_{\text {observed }}=2 \mu_{0} n \kappa T / B_{z}^{2}=4 \times 10^{-4}$ which is very close to $\beta_{\text {predicted }}$. If the model were wrong, one would expect no relationship between the predicted and observed $\beta$ 's, i.e., one would expect a discrepancy of many orders of magnitude between the predicted and the observed $\beta$ 's.

This model also has implications regarding the brightening typically observed when the axis of a coronal loop starts to writhe and the loop develops a kink instability (sigmoid). Since kink instability occurs when $\alpha h \sim 1$ and for a long thin flux tube $a<<h$, this model predicts that $\beta=\alpha^{2} a^{2} / 2<<\alpha^{2} h^{2} / 2$ will still be small even if $\alpha$ is increased to the point where $\alpha h \sim 1$ and kink instability occurs. However, $\beta$ will increase as $\alpha$ increases and so this model predicts that the loop should brighten in proportion to the writhing of its axis (i.e., in proportion to $\alpha$ as $\alpha h$ approaches unity).

Finally, we note that Feldman (2002) has recently used purely observational evidence to argue that electric currents with geometry similar to what is discussed here are the means by which the Sun and similar stars produce their coronal activity.

Acknowledgment: Supported by USDOE Grant DE-FG03-97ER54438.

\section{REFERENCES}

Aschwanden, M. J., Nightingale, R. W., and Alexander, D. 2000, ApJ 541, 1059

Bellan, P. M., Spheromaks (Imperial College Press, 2000, London)

Chen, F. F., Introduction to Plasma Physics and Controlled Fusion (Plenum 1984, New York)

Feldman, U. 2002, Physica Scripta 65, 1985

Klimchuk, J. A. 2000, Solar Physics 193, 53

Pevtsov, A. A., Canfield, R. C., and McClymont, A. N. 1997, ApJ 481, 973

Polygiannakis, J. M. and Moussas, X. 1999, Plasma Phys. Control. Fusion 41, 967

Uchida Y. and Shibata, K. 1988, Solar Phys. 116, 291 